\title{
JURISPRUDENCIA AMBIENTAL EN ARAGÓN (PRIMER SEMESTRE 2018)
}

\author{
Laura Salamero Teixidó \\ Profesora ayudante doctora \\ Universidad de Lleida
}


Sumario: 1. Suspensión de algunos preceptos del plan general de pesca de Aragón para el año 2018. 2. La siempre difícil convivencia entre la actividad ganadera y los núcleos de población. 3. Acerca de la necesaria declaración de impacto ambiental para la apertura de una pista forestal. 4. Consecuencias de la falsedad del contrato tipo en el otorgamiento de subvenciones. 5. Sobre el concepto de interesado en un procedimiento de concesión de aprovechamiento de aguas públicas. 6. EI TSJ confirma la nulidad de algunos preceptos de la Ordenanza Municipal reguladora de la Instalación y del Funcionamiento de Infraestructuras Radioeléctricas de Huesca. 7. Sobre la renovación de una autorización del Sistema Colectivo de Neumáticos fuera de Uso y su conjugación con la normativa básica estatal en la materia.

\section{SUSPENSIÓN DE ALGUNOS PRECEPTOS DEL PLAN GENERAL DE PESCA DE ARAGÓN PARA EL AÑO 2018}

Mediante dos autos de 21 de febrero y de 7 de marzo, el Tribunal Superior de Justicia de Aragón acuerda y mantiene respectivamente la suspensión provisional de determinados preceptos del Plan General de Pesca de Aragón para el año 2018.

La adopción de la medida cautelar de suspensión se da en el marco del recurso directo interpuesto por la Federación Aragonesa de Pesca y Casting contra la Orden DRS/202/2018, de 31 de enero, por la que se aprueba el Plan General de Pesca de Aragón para el año 2018, publicada en el Boletín Oficial de Aragón (BOA) con fecha 14/02/2018. En dicho recurso se solicitó como medida cautelarísima y, subsidiariamente, como medida cautelar ordinaria, con carácter principal, la suspensión de la vigencia de la Orden y, con carácter subsidiario, la suspensión de la vigencia de la Orden impugnada relativa a los siguientes preceptos y disposiciones: a. La inclusión en el artículo 2.2 de la trucha arcoíris, la carpa común y el black bass, pasando a regularse conforme al art. 2.1 como especies pescables, en la modalidad de captura y suelta (sin muerte). b. Los artículos 2.5; 4.3; 14.1; 14.2, apartados a), c), d) y e); 14.3; 15; 28.4.b); 33.1; $36.1 ; 36.2$ y el Anexo XII, porque alteran el sistema tradicional de captura y suelta obligando al pescador a sacrificar inmediatamente y por el medio especificado los ejemplares capturados de trucha arcoíris, carpa común y black bass, debiendo eliminarlos del medio natural.

\section{LA SIEMPRE DIFÍCIL CONVIVENCIA ENTRE LA ACTIVIDAD GANADERA Y LOS NÚCLEOS DE POBLACIÓN.}

El titular de una explotación porcina ubicada en Bierge (Huesca), solicitó el cambio de orientación productiva de dicha explotación. De la denegación de tal solicitud, trae causa la sentencia de la sección $1^{\text {a }}$ de la Sala de lo Contencioso- 
Administrativo del TSJ aragonés 15/2018 de 18 de enero (recurso de apelación núm. 319/2015).

La petición del recurrente fue desestimada por la Administración de la Diputación General de Aragón en atención a los siguientes fundamentos legales. Resulta de aplicación al caso el Decreto 94/2009, de 26 de mayo, del Gobierno de Aragón, por el que se aprueba la revisión de las Directrices sectoriales sobre actividades e instalaciones ganaderas, en cuyo artículo 21 regulan las distancias mínimas que deben guardarse entre las instalaciones ganaderas y los núcleos de población. $Y$ en concreto interesa resaltar los apartados segundo y séptimo de dicho precepto.

De un lado, según el art. 21.2: «Las distancias a los núcleos de población en los municipios que cuenten con alguna figura de planeamiento aprobada o de delimitación de suelo urbano, se medirán desde el punto de la línea de delimitación de los suelos urbanizable o urbano del núcleo de población más próximo a la explotación ganadera hasta el punto más próximo construido de la explotación ganadera, sean los edificios u otros elementos funcionales de la misma susceptible de producir emisiones molestas, nocivas, o insalubres».

Del otro, según el art. 21.7: «Sin perjuicio de lo dispuesto en la normativa estatal, las distancias a los suelos urbanos o urbanizables de los núcleos de población podrán reducirse hasta la mitad, por Acuerdo del Pleno Municipal, en circunstancias excepcionales, en municipios enclavados en Zonas desfavorecidas de montaña, de acuerdo con la Directiva del Consejo 86/466/CEE, de 14 de julio de 1986, y la Decisión 89/566/CEE, de 16 de octubre, todo ello según los criterios de Delimitación de Zonas desfavorecidas establecidos en el artículo 3, apartado 3, de la Directiva 75/268/CEE, constando en el Anexo IX la relación de Municipios enclavados en dichas zonas. Cuando la reglamentación técnico sanitaria establezca una distancia mínima no será posible la reducción de ésta. No podrá acordarse reducción alguna para los siguientes casos: a) Explotaciones de porcino. (...)».

En atención dichos fundamentos legales, la solicitud y recursos del recurrente son desestimados. De un lado, porque su instalación porcina se halla a una distancia de 7.30 metros del núcleo urbano. Al existir en el municipio de Bierge 
un Proyecto de Delimitación de Núcleo Urbano aprobado por la Comisión de Ordenación del Territorio en 2000 y 2003, no pueden tenerse en cuenta para el cálculo de las distancias mínimas otros elementos, como reclama el recurrente.

Asimismo, tampoco es de aplicación al recurrente la reducción del $50 \%$ de la distancia mínima, puesto que dicha reducción está excluida de las explotaciones porcinas, como es el caso.

Por todo ello, el tribunal resuelve la desestimación del recurso de apelación presentado.

\section{ACERCA DE LA NECESARIA DECLARACIÓN DE IMPACTO AMBIENTAL PARA LA APERTURA DE UNA PISTA FORESTAL.}

La sentencia que se comenta trae causa de la Resolución de la Directora del Instituto Aragonés de Gestión Ambiental de 2 de diciembre de 2013 por la que se decide no someter al procedimiento de evaluación ambiental el proyecto de apertura de accesos desde el núcleo de "La Cabezonada" a la zona de pastos de "La Estiva" en el término municipal de La Fueva (Huesca), promovido por los propietarios forestales de La Cabezonada.

Tal decisión administrativa se fundamenta en el argumento de que el proyecto no se encuentra expresamente recogido en el Anexo II de la Ley 7/2006, de 22 de junio, de Protección ambiental de Aragón, lo que determinaría según su art. 24.1 la obligación de sometimiento a evaluación de impacto ambiental. Por el contrario se considera que está incluida en el supuesto 9.6 del Anexo III, en cuyo caso, según el art. 24.2 de la misma Ley «solo debe someterse a evaluación cuando así lo decida el órgano ambiental en cada caso». En este caso, el órgano competente no lo consideró conveniente. Téngase en cuenta que la Ley 7/2006 fue derogada por la Ley 11/2014, de 4 de diciembre, de Prevención y Protección Ambiental de Aragón.

Los recurrentes exigen la inclusión de la evaluación ambiental en el proyecto mentado. En primer lugar porque consideran que el proyecto se trata de uno de los previstos en el Grupo 10.2 del Anexo II de la Ley $7 / 2006$, que hubiera obligado según el art. 24.1 el sometimiento a evaluación de impacto ambiental. En el mentado Grupo 10.2 se incluyen proyectos que implican transformaciones de uso del suelo que impliquen la eliminación de la cubierta vegetal cuando dichas 
transformaciones afecten a superficies superiores a 10 hectáreas que se desarrollen en zonas designadas en aplicación de la Directiva 79/409/CEE, del Consejo, de 2 de abril, relativa a la conservación de las aves silvestres, y de la Directiva 92/43/CEE, del Consejo, de 21 de mayo, relativa a la conservación de los hábitats naturales y de la fauna y flora silvestres, o en humedales incluidos en la lista del Convenio de Ramsar. En la sentencia dictada en primera instancia que ahora se recurre en apelación se razonó que a pesar de que la pista supera la superficie de 10 hectáreas, la pista afecta a una superficie menor que no existe transformación del uso del suelo.

Ante los anteriores argumentos, la sección 1a de la Sala de lo ContenciosoAdministrativo del Tribunal Superior de Aragón, en la sentencia 22/2018, de 24 de enero (recurso de apelación 116/2016), estima que debería de forma obligatoria tramitarse la evaluación de impacto ambiental de un lado, porque la construcción de la pista forestal implica una transformación del suelo y, del otro, porque, de conformidad con la pericial presentada, la superficie afectada supera las 10 hectáreas. Todo ello, en atención al principio de precaución o cautela que debe regir la interpretación de las normas en materia de protección del medio ambiente. Así, se estima el recurso de apelación, retrotrayéndose las actuaciones e imponiendo la obligación de realizar la evaluación de impacto ambiental para autorizar la pista forestal.

\section{CONSECUENCIAS DE LA FALSEDAD DEL CONTRATO TIPO EN EL OTORGAMIENTO DE SUBVENCIONES.}

Durante el periodo analizado el TSJ ha dictado varias sentencias sobre esta misma cuestión (sentencias 31/2018, de 31 de enero, y 45/2018, 50/2018 y $51 / 2018$ todas ellas de 6 de febrero).

Todas las sentencias mentadas traen causa de sucesos idénticos. Tras la entrada en vigor de la reforma de la Política Agraria Común conocida como «chequeo médico», regulada en el Reglamento CE 73/2009, se integraron en el régimen de pago único determinadas ayudas que no se habían incorporado en el año 2006. De ahí que se incrementara el valor de los derechos que tenían o se generaran nuevos para aquellos agricultores que en las campañas 2007/2008 y 2008/2009 -entre otros-: hayan participado en el régimen de ayuda a la transformación de forrajes desecados. 
En atención a ello, el Consejero de Agricultura, Ganadería y Medio Ambiente aragonés resolvió según los datos que constaban en un contrato tipo a disposición de la Administración entre el agricultor y la empresa transformadora de forrajes. Resultó, no obstante, que la firma del agricultor que constaba en dicho contrato había sido falseada. El Tribunal entiende que de tal vicio contractual se deriva la nulidad de la resolución administrativa. EI TSJ estima que es capaz de valorar la nulidad del contrato derivada de la falsificación de una de las firmas en una cuestión prejudicial civil y ello le permite estimar el recurso. Ello implica una mejora sustancial de la ayuda a recibir por el recurrente.

\section{SOBRE EL CONCEPTO DE INTERESADO EN UN PROCEDIMIENTO DE CONCESIÓN DE APROVECHAMIENTO DE AGUAS PÚBLICAS.}

En la sentencia 390/2017, de 14 de noviembre (recurso 75/2016), el TSJ de Aragón resuelve a favor del recurrente que reclamaba la nulidad de un procedimiento administrativo de modificación de la concesión de aprovechamiento de aguas públicas resuelto por la $\mathrm{CHE}$, puesto que como interesado necesario en este, no fue llamado y no pudo defender sus intereses en el mismo. Siguiendo una línea e interpretación jurisprudencial consolidada, el TSJ acoge la pretensión del recurrente no solo porque los datos del recurrente constaban en el expediente administrativo de marras, sino también porque entiende que este, como titular de un aprovechamiento colindante, tiene un claro interés en el procedimiento antes mencionado. Por todo ello, declara la nulidad de la resolución y ordena la retroacción de las actuaciones.

\section{EL TSJ CONFIRMA LA NULIDAD DE ALGUNOS PRECEPTOS DE LA ORDENANZA MUNICIPAL REGULADORA DE LA INSTALACIÓN Y DEL FUNCIONAMIENTO DE INFRAESTRUCTURAS RADIOELÉCTRICAS DE HUESCA.}

El Pleno del Ayuntamiento de Huesca modificó la Ordenanza reguladora de la Instalación y del Funcionamiento de Infraestructuras Radioeléctricas, publicada en el Boletín Oficial de la Provincia de Huesca de 4 de septiembre de 2013. France Telecom España S.A, impugnó la modificación de la Ordenanza en tanto que entiende que el Ayuntamiento se extralimita en el ejercicio de su competencia normativa, vulnerando además lo que se establece en la Ley 12/2012, de 26 de diciembre, de medidas urgentes de liberalización del comercio y determinados servicios. 
El TSJ de Aragón, en sentencia 92/2018, de 23 de enero (recurso núm. 234/2013) estima algunas de las pretensiones de la demandante, en concreto aquellas relativas a los criterios para la instalación de equipos.

En concreto el texto de la ordenanza según la modificación rezaba del siguiente modo: «3. Se procurarán aplicar los umbrales recomendados y todo lo indicado en las recomendaciones, tanto a nivel estatal como internacional, y, más en concreto, a lo indicado en la resolución número 1815, de 27 de mayo de 2011 de la Asamblea Parlamentaria del Consejo de Europa. Aquellos casos en los que no sea posible cumplir este requisito deberá justificarse, presentando documentación al respecto. 4. De manera análoga al anterior punto, se procurarán aplicar las distintas recomendaciones y normativas respecto a la ubicación, características y condiciones de funcionamiento de las estaciones radioeléctricas para minimizar los niveles de emisión sobre espacios sensibles, como escuelas, centros de salud, hospitales, parques públicos, residencias, institutos, etc.). Aquellos casos en los que no sea posible cumplir este requisito deberá justificarse, presentando documentación al respecto».

Según el TSJ: «Examinado el tenor literal de los apartados impugnados, podrá comprobarse que la remisión concreta a la Recomendación de la Asamblea Parlamentaria del Consejo de Europa a la que hace expresa referencia, determina la asunción por la Ordenanza, normativizándola, de unos concretos umbrales de emisión de radiaciones que vienen establecidos en la Recomendación y que, hasta ese momento, carecen de fuerza normativa. En definitiva, que la regulación municipal impugnada, aunque expresada en términos tan débiles como se plasman en dichos apartados “...se procurará...", excede sobradamente y puede encontrar difícil justificación en competencias municipales como las invocadas. De un modo u otro, con mayor o menor intensidad, se introducen nuevos umbrales de emisión de radiaciones que excede de las propias competencias para adentrarse en terreno de exclusiva competencia estatal, tal y como la Sala Tercera del Tribunal Supremo ha tenido ocasión de tratar ya en la antedicha sentencia, reproducida parcialmente en el escrito de demanda». Por todo ello, se estima el recurso y declara la nulidad de los preceptos transcritos. 


\section{SOBRE LA RENOVACIÓN DE UNA AUTORIZACIÓN DEL SISTEMA COLECTIVO DE NEUMÁTICOS FUERA DE USO Y SU CONJUGACIÓN CON LA NORMATIVA BÁSICA ESTATAL EN LA MATERIA.}

La sentencia que se analiza trae causa de la Resolución del Presidente del INAGA de 12 de marzo de 2015, por la que se estimaba parcialmente el recurso de alzada interpuesto frente a la Resolución de la Directora del INAGA de 19 de marzo de 2014, que renovaba la autorización del Sistema Colectivo de Neumáticos fuera de Uso, para todo el ámbito territorial de la Comunidad Autónoma de Aragón, dejando sin efecto la resolución de 27 de abril de 2007 de la Dirección General de Calidad Ambiental.

Dicha resolución de 2015 fue recurrida en sede contencioso-administrativa por Tratamiento De Neumáticos Usados, S.L., resultando estimado el recurso de modo que tal resolución del INAGA fue anulada. Los argumentos de la sentencia de instancia fueron que mediante dicha Resolución el INAGA «va más allá en el ejercicio de competencias autonómicas que, siendo cierto que son propias de la Comunidad Autónoma, sin embargo no han sido objeto de desarrollo normativo ni habilitación legal ninguna, de suerte que, en consecuencia, aplicando la doctrina que deriva de la sentencia de la Sala Tercera de 10 de diciembre de 2009, la Comunidad Autónoma, en la resolución impugnada, está adoptando medidas adicionales a las derivadas de la normativa básica estatal y reglamentaria aplicable, y estableciendo medidas adicionales que implican nuevas responsabilidades de los afectados o un régimen de responsabilidad más intenso, que deben tener un amparo normativo que asegure la legalidad de las mismas. No consta que la Comunidad Autónoma de Aragón, haya desarrollado sus competencias en esta materia. De este modo, el acto administrativo impugnado rebasa indebidamente la normativa aplicable. Las competencias que en materia de protección del medio ambiente se atribuye a las Comunidades Autónomas, necesitan de una previa habilitación legal, si se dirigen, como en el caso presente a criterio de la Juez de instancia, a restringir las actuaciones de terceros, habilitación legal que, como se ha dicho, no existe en este caso por falta de desarrollo normativo de la propia competencia autonómica. Que se actúe en defensa de intereses colectivos no es suficiente para legitimar la actuación impugnada, siendo preciso un desarrollo normativo previo de la propia competencia, que no existe en este caso». 
EI TSJ de Aragón, mediante la sentencia 99/2018, de 2 de marzo (recurso de apelación 104/2016), recoge los argumentos de la parte recurrente, el INAGA y estima que la Resolución de renovación de la autorización del Sistema Colectivo de Neumáticos fuera de Uso era ajustada a Derecho. En concreto los fundamentos del a sentencia son los siguientes: «Si bien es cierto que, efectivamente, las competencias que en materia de protección del medio ambiente se atribuyen a las Comunidades Autónomas, por las que se les habilitan potestades de diferente naturaleza, precisan de una previa habilitación legal y desarrollo normativo si se dirigen a restringir la actividad de los interesados, a agravar o empeorar la situación de la entidad o sujeto autorizado, por referencia a la derivada de la estricta aplicación de la normativa básica estatal, sin embargo no compartimos con la Juez de instancia el hecho que da por cierto y presupone, por el cual la autorización impugnada esté agravando la situación derivada de la estricta aplicación de la normativa básica estatal, única aplicable al presente supuesto por ausencia de desarrollo normativo por la Comunidad Autónoma». Y, examinando el texto en cuestión en TSJ entiende que «(...) con la resolución impugnada, no se agrava la situación de la entidad autorizada, ni se actúan competencias no habilitadas ni desarrolladas normativamente, sino que se limita a centrar, precisamente, el terreno de ejercicio de las competencias que la propia normativa básica confiere». 